\title{
Research Progress on Physical Storage Methods of Toona Sinensis
}

\author{
Li Song $^{1 *}$, Li Benjiao ${ }^{1}$, Ma Binrong ${ }^{1}$, Jing Yong ${ }^{1}$, Wang Qiang ${ }^{1}$, Zhang Zheming $^{1}$ \\ ${ }^{1}$ Agricultural Science Institute of Dazhou, Dazhou, 635000, China
}

\begin{abstract}
The toona sinensis (or Chinese toon) in rich in nutrition, with unique aroma and broad prospects for use. However, the toona sinensis is prone to respiration after being harvested. As a heat-sensitive vegetable, it is easy to lose water and decay. This paper introduced the latest research progress on physical preservation of toona sinensis before and during storage, providing references for developing new technologies of keeping Chinese toon fresh.
\end{abstract}

\section{Introduction}

Toona sinensis, a perennial deciduous tree of Meliaceae, is categorized as woody vegetable that is unique in China. There are 4 varieties and 6 variants in China, which are distributed in south, southwest and North China. Toona sinensis feature strong adaptability, fast growth and functioning as food, wood, medicine and ornamental plants ${ }^{[1]}$. However, toona sinensis is a heatsensitive vegetable, characterized by strong seasonality, fresh texture, high water content and vigorous respiration. After harvesting, at temperature higher than $10^{\circ} \mathrm{C}$, it is easy to deteriorate, defoliate and rot ${ }^{[2]}$, with changes or even disappearance of its original flavor. When it is left at room temperature for about 6 days, it loses almost all its edible values ${ }^{[3]}$. Recently, there have been researches on the preservation of toona sinensis. Compared with chemical and biological preservation, physical preservation of toona sinensis features accessibility, efficiency and no chemical residue, and has therefore attracted attention from a growing number of researchers. This paper presented the latest progress on physical preservation of toona sinensis, laying foundation for further technical promotion and application.

Table 1. Varieties anddistributions of Toona sinensis.

\begin{tabular}{|c|c|}
\hline Varieties & Distributions \\
\hline $\begin{array}{c}\text { Toona ciliata } \\
\text { Roem }\end{array}$ & $\begin{array}{c}\text { Fujian, Hunan, Guangdong, } \\
\text { Guangxi, Sichuan, Yunnan, etc }\end{array}$ \\
\hline $\begin{array}{c}\text { Toona microcarpa } \\
\text { (C. DC.) C. Y. Wu }\end{array}$ & $\begin{array}{c}\text { Hubei, Guangdong, Hainan, } \\
\text { Guangxi, Yunnan, etc }\end{array}$ \\
\hline $\begin{array}{c}\text { Toona rubriflora } \\
\text { Tseng }\end{array}$ & $\begin{array}{c}\text { Nanjing and Yongding in Fujian } \\
\text { Province }\end{array}$ \\
\hline $\begin{array}{c}\text { Toona sinensis (A. } \\
\text { Juss.) Roem }\end{array}$ & Shandong, Henan, Hebei \\
\hline
\end{tabular}

\footnotetext{
* Corresponding author: 306368980@qq.com
}

\section{Effect of pre-treatment on the preservation of toona sinensis}

\subsection{Cleaning}

Cleaning is an essential step for the preservation and processing of fresh vegetables. At present, many developed countries have taken the cleaning and sterilization of vegetables as a critical control point of hazard analysis (HACCP). Good cleaning treatment can prevent diseases caused by pathogenic bacteria and prolong the storage of vegetables ${ }^{[4,5]}$. Zhu Miao et al. ${ }^{[6]}$ compared the effects of deionized water, sodium benzoate, hydrogen peroxide, sodium hypochlorite and ultrasonic cleaning on the quality of fresh toona sinensis during storage. Although different cleaning methods have different mechanisms, they have no significant effect on soluble protein content, respiratory intensity and weight loss rate of fresh toona sinensis during storage. Hydrogen peroxide and sodium hypochlorite containing $0.01 \%$ active chlorine have a better performance in the preservation of fresh fruit, but hydrogen peroxide could seriously damage the sensory and nutritional components of toona sinensis. Sodium hypochlorite containing active chlorine can better maintain the content of total sugar, vitamin $\mathrm{C}$ and leaf of fresh toona sinensis, and prolong the storage of fresh toona sinensis to 12 days. Therefore, sodium hypochlorite with $0.01 \%$ active chlorine is the most suitable cleaning agent for pretreatment.

\subsection{Sterilization}

Given toona sinensis is heat-sensitive, there are many drawbacks in the traditional thermal sterilization of toona sinensis. Heating treatment changes the chemical composition of toona sinensis, destroys its unique flavor, 
and seriously damages its nutritional values ${ }^{[7]}$. Zhou Jianmei ${ }^{[8]}$, Bao Lin et al. ${ }^{[9]}$ used $95^{\circ} \mathrm{C}$ perm as the pretreatment method of frozen toona sinensis. It was found that the hot drift process caused more nutritional losses of Toona sinensis, especially Vc, but POD activity was inhibited, and the rate of $\mathrm{Vc}$ decreased during freezing. Chen Lijuan et al. ${ }^{[10]}$ studied the effect of hot float time on the quality of toonaona sinensis in boiling water. It was found that long-term hot drift decreased the polyphenols content in the tender leaves of Toona sinensis, and the relationship decreased linearly from 0 to $1.0 \mathrm{~min}$. The content of flavonoids decreased sharply from 0.5 to $1.0 \mathrm{~min}$ in hot floats; the effect of perming float on soluble proteins was not obvious. The activity of the first $0.5 \mathrm{~min}$ nitrite and PPO decreased sharply, and the hot float $0.5 \mathrm{~min}$ was considered as a more suitable treatment time. Yang Hui et al. ${ }^{[11]}$ studied the effect of hot drift on the volatile components of Toona sinensis. The results showed that after the increase of the variety of volatile ingredients in Toona sinensis, the decrease of irritating ingredients such as sulfur, the increase of terpenoid soft gases, and to a certain extent, the aroma has increased.

Some scholars studied the cold sterilization of toona sinensis, and found the utilization of specific sterilization method has an important impact on toona sinensis, causing different levels of spoilage and deterioration of the vegetable during storage. Zhao Fang et al. ${ }^{[12]}$ treated toona sinensis with ultra-high pressure, ultraviolet ray and ozone respectively, and observed similar changes in toona sinensis. Ultra-high pressure can effectively kill microorganisms, with the highest sterilization rate of 98.44\% and the vitamin $\mathrm{C}$ preservation of $78.57 \%$ respectively. Ultraviolet sterilization kept the most soluble sugar in toona sinensis during storage, with a content of $86.96 \%$. Ozone sterilization significantly reduced chlorophyll and vitamin $\mathrm{C}$ of toona sinensis during storage. Given the growth rate of toona sinensis colonies and the sensory scores, the ultra-high pressure treatment had the best performance, with the flavor and nutritional components of toona sinensis being kept well.

\section{Effect of storage conditions on preservation of toona sinensis}

\subsection{Temperature and humidity}

Water content is an important measurement of the freshness of toona sinensis. Toona sinensis has a high level of water content when it is just picked. Transpiration and respiration could lower nutrients and water in toona sinensis ${ }^{[13]}$. Shui Jiangbo et al. ${ }^{[14]}$ studied the transpiration and respiration of toona sinensis by adjusting the temperature and humidity, with the weight loss rate, vitamin $\mathrm{C}$ and chlorophyll as indicators. The results showed that the loss rate of weight, vitamin $\mathrm{C}$ and chlorophyll was the lowest at $1^{\circ} \mathrm{C}$ and $95 \%$ humidity. Toona sinensis could be well stored at low temperature and high humidity as low temperature can reduce the activity of respiratory enzymes and lower respiratory intensity, while high humidity can adjust the osmotic pressure inside and outside toona sinensis tissues, hindering water release between cells and tissues, and thus effectively reducing the transpiration of toona sinensis.

Zhao Hua et al. ${ }^{[15]}$ found toona sinensis showed aging symptoms at about $2 \mathrm{~d}$ below $20^{\circ} \mathrm{C}$, and the leaves began to fall off, with a drop rate of $98 \%$ at $5 \mathrm{~d}$. At $10^{\circ} \mathrm{C}$, the leaves began to fall off at about $5^{\circ} \mathrm{C}, 67 \%$ at $11 \mathrm{~d}$, and $20 \mathrm{~d}$ at $5^{\circ} \mathrm{C}$ and $29 \mathrm{~d}$ under $0{ }^{\circ} \mathrm{C}$, both less than $1 \%$, but there was a small amount of decay or darkening of color. Du Jian et al. ${ }^{[16]}$ explored the changes of polyphenol antioxidant after the storage of Toona sinensis at a certain time of 0,5 and $10^{\circ} \mathrm{C}$, and found that $0{ }^{\circ} \mathrm{C}$ storage has a strong free radical removal capacity compared with 5 and $10{ }^{\circ} \mathrm{C}$. This is consistent with studies such as Yang Ying et al. [17]. Fresh Toona sinensis leaves were stored at $0,5,10$ and $20{ }^{\circ} \mathrm{C}$, with the longest storage time and the slowest physiological changes at $0{ }^{\circ} \mathrm{C}$, and the activity changes of polyphenol content and polyphenol oxidase and peroxidase enzymes are the smoothest.

\subsection{Compression}

Decompression storage is a controlled atmosphere storage developed on the basis of refrigeration. Decompression treatment reduces the partial pressure of oxygen and other gas inside the container, thus playing a role similar to refrigeration combined with modified atmosphere storage. Wang Zhaogai et al. ${ }^{[18]}$ studied the effects of "(A) constant pressure of $20 \mathrm{kPa}$ " and "(B) $20 \mathrm{kPa} \rightarrow$ normal pressure $\rightarrow 60 \mathrm{kPa}$ " on the storage of red oil toona sinensis seed bud. The results showed that reduced pressure treatment could effectively inhibit the respiratory intensity of toona sinensis, significantly reduce the rate of defoliation, decay and weight loss, greatly slow down the loss of vitamin $\mathrm{C}$ and chlorophyll, and prevent the activity of polyphenol oxidase. Meanwhile, Plan B had a better performance than Plan A.

\subsection{Packaging materials}

Active modified atmosphere packaging for fresh fruits and vegetables can not only reduce transpiration and water loss of fruits and vegetables after harvest, but also adjust the proportion of $\mathrm{O}_{2}$ and $\mathrm{CO}_{2}$ in the bag through respiration of fruits and vegetables and the air permeability of packaging bags, thus inhibiting respiration and other physiological metabolism and keeping fruits and vegetables fresh. In addition, it can also reduce the infection of pathogenic bacteria and decay loss, featuring a simple, economically efficient and highly effective method for preservation of postharvest fruits and vegetables ${ }^{[19-21]}$. Zhu Yongqing ${ }^{[22]}$ and others used low-density polyethylene (LDPE) and high-density polyethylene (HDPE) at the thicknesses of 0.01 and $0.03 \mathrm{~mm}$ as materials for active modified atmosphere packaging to store "Bashanhong" toona sinensis produced in Dazhu County, Dazhou, Sichuan Province. The results showed that all the four packaging 
treatments could reduce weight loss, defoliation, cell membrane permeability and nitrite content of toona sinensis, while LDPE (with the thickness of $0.03 \mathrm{~mm}$ ) was better in keeping the color of toona sinensis, and could effectively prolong the preservation of toona sinensis to 25 days at $4^{\circ} \mathrm{C}$.

\section{Mixed preservation techniques}

After toona sinensis is harvested, treatment before and during storage, and post-storage transportation can all affect the quality of toona sinensis. Limitations in the existing fresh-keeping techniques cannot yet meet the needs of consumers. Therefore, there are scholars using compound preservation techniques to maximize the quality of toona sinensis after storage. Wang Liqiong et al. [23] compared the effects of three fresh-keeping methods of PVC combined with ultraviolet radiation, PVC combined with 1-methylcyclopropene and PVC combined with ethylene absorbent on the storage of toona sinensis. All the three preservation methods displayed preservation functions, among which PVC combined with UV radiation can significantly delay the change of color and the loss of weight, boost chlorophyll, inhibit respiration, ethylene release and polyphenol oxidase activity, and improve the activities of peroxidase, catalase and peroxidase, exhibiting the best preservation performance.

Yang Hui et al. ${ }^{[24]}$ studied the storage of toona sinensis buds using opening and humidity control packaging at ice temperature. After precooling the buds at $4^{\circ} \mathrm{C}$ for 12 hours, they were packed into PE freshkeeping bags with different opening areas (at the thickness of $0.06 \mathrm{~mm}$ ), and then sealed and stored in warehouse at ice temperature $\left(-0.5^{\circ} \mathrm{C}\right)$. At $-0.5^{\circ} \mathrm{C}$, with an opening area of $141.13 \mathrm{~mm} 2$ for the PE freshkeeping bag (accounting for $0.14 \%$ of the total area), it can effectively reduce the weight loss rate of the buds, delay the decline of $\mathrm{VC}$, soluble protein, total polyphenols, soluble sugar, chlorophyll and flavonoids, slow down the process of membrane lipid peroxidation, inhibit the activity of polyphenol oxidase, and better maintain the quality of toona sinensis buds during storage.

Ma Jiajia et al. [25] discussed the effect of microporous packaging and low temperature plasma sterilization on the storage of toona sinensis on shelf. Using microporous packaging alone (pore size: $100 \mu \mathrm{m}$, number of pores: 11), low-temperature plasma cold sterilization alone (voltage: $45 \mathrm{kV}$, time: $50 \mathrm{~s}$, polar distance: $60 \mathrm{~mm}$ ) and mixed treatment, the storage was simulated on shelf (temperature: $10^{\circ} \mathrm{C}$, humidity: $75 \%$ ). The results showed that the mixed treatment integrating microporous packaging and cold sterilization can significantly delay the deterioration of toona sinensis, inhibit the decrease of $\alpha$ value and the increase of shear force of young stems, while microporous packaging can delay the decay of toona sinensis, maintain a high sensory score and slow down the increase of nitrite content. Principal component analysis showed that the variance contribution rate reached $95.28 \%$, and toona sinensis stored for 8 days could be clearly distinguished. For the variables of storage time and treatments, the total contribution rate of linear discriminant analysis was only $60.73 \%$. Microporous packaging and low-temperature plasma sterilization can delay the deterioration of toona sinensis, keep its appearance and flavor, and maintain a shelf life of 4 - 6 days.

\section{Prospect}

Toona sinensis is a specialty of Dazhu County, Dazhou City, Sichuan Province, and a national geographical indication product of China ${ }^{[26-27]}$. Daylighting in Dazhu County is suitable, with abundant rainfall and a long frost-free period, which is very suitable for the growth of Toona sinensis. Dazhuxiang is a pure natural green food because it does not apply fertilizer and does not use pesticides. It sells well in more than 20 large and medium-sized cities in China with its unique qualities such as smooth leaves, bright red color and unique aroma.

Toona sinensis, as a woody vegetable, has high economic value, and prolonging the freshness of toona sinensis is an important means to improve the economic value of toona sinensis. Nutrition loss, browning and decay are common forms of quality deterioration in the storage process of Toona sinensis, This leads to a short sales cycle and high price fluctuations. At present, there are relatively few studies on the preservation technology of ton after toona sinensis in foreign countries, mainly by domestic scholars, mostly focusing on macroscopic effects, and lack of in-depth mechanism research.

Compared with chemical and biological preservation, the physical storage of toona sinensis features wider choices of storage and application, enhanced food safety, and more effective storage before, during and after the harvest of toona sinensis. However, the heat-sensitive property has prevented toona sinensis from being stored for a long time, thus hindering the development of the toona sinensis industry. Therefore, it is necessary to find novel and more effective physical preservation methods for toona sinensis. It is recommended to integrate physical preservation with chemistry and biology preservation to prolong the storage of toona sinensis as soon as possible.

\section{References}

1. Liu Changjin. Toona Sinensis [M]. Tianjin: Tianjin Science and Technology Press, 2016: 116-121.

2. Dai Guizhi. Toona Sinensis Stored for Consumption Out of Season [J]. Northern Horticulture, 2006 (5): 175-176.

3. Zhang Heyi. Preservation and Processing of Toona Sinensis [J]. Preservation and Processing, 2003, 3 (5): 9-13.

4. Wang Wenxian, Xie Yonghong, Liu Xuewen et al. Study on the Preservation of Toona Sinensis Buds [J]. Food Science and Technology, 2007, 31 (10): 218-220. 
5. Zhao Qin. Progress on Storage and Preservation of Woody Vegetable Toona Sinensis Buds [J]. Guizhou Forestry Science and Technology, 2008, 36 (3): 55-58.

6. Zhu Miao, Lu Qingsong, Li Gangfeng et al. Effects of Different Cleaning Methods on the Quality of Fresh Toona Sinensis Buds during Storage [J]. Northern Horticultural Science, 2016 (21):136-139.

7. Li Nan, Wang Haoyu, Zhao Fang, et al. Effects of Sulfur Compounds in Toona Sinensis Sprouts on Their flavor $[\mathrm{J}]$. Food Research and Development, 2017, 38 (13): 35-39.

8. Zhou Jianmei, Wang Chengnan, Liu Bin, etc. Fastfrozen preservation and dehydration processing technology of fennel buds [J]. Economic Forest Research, 2011, 29 (2): 101-103.

9. Bao Lin,Yuan Yuchao. Effect of hot bleaching and frozen time on the quality of frozen fennel $[\mathrm{J}]$. Food Technology, 2007 ( 9) : 76-78.

10. Chen Lijuan, Wang Zhao, Yang Hui, etc. Study on the effect of bleaching time and storage temperature on the quality of fennel buds [J]. Food Research and Development, 2016, 37 (2): 19-23.

11. Yang Hui, Wang Zhao, Shi Guanying, etc. Study on the effect of hot-floating time on the color and volatile flavor composition of fennel buds [J]. Journal of Nuclear Agriculture, 2017, 31 ( 7) : 1339 -1348 .

12. Zhao Fang, Sun Xiaojian, Yu Pengfei et al. Effects of Different Cold Sterilization Techniques on the Quality of Toona Sinensis during Storage [J]. Food Research and Development, 2018, 39 (4): 187-192.

13. Zhang Xiangmei, Zhao Fengcun, Li Huili, et al. The Effect of Small Root Garlic Extract on the Preservation of Toona Sinensis [J]. Chinese Agricultural Science Bulletin, 2009, 25 (4): 55-58.

14. Shui Jiangbo, Miao Miao, Ren Meng, etc. Impact of Different Temperature and Humidity on the Preservation of Toona Sinensis [J]. Home Appliances Science and Technology, 2016 (10): 8183.

15. Zhao Hua, Hu Hong, Wu Zhaozhi, etc. Effect of storage conditions on the shedding of fennel bud leaves [J]. Acta Horticulturae Sinica, 1997 ( 3) : 5458

16. Du Jian, Yang Ying, Zhao Mingxuan, etc. Study on changes in the antioxidant properties of fennel polyphenols in storage [J]. Beijing Forestry University, 2011, 33 ( 2) : 120-125.

17. Yang Ying, Zhai Zhien, Wang Jun, etc. Relationship between polyphenol content and associated enzyme activity changes in fennel during storage [J]. Food Technology, 2010, 35 ( 2) : 24-28.

18. Wang Zhaogai, Yang Hui, Zhu Guangcheng, et al. Study on the Effect of Decompression on the Storage of Toona Sinensis [J]. Acta Agriculturae Boreali-Sinica. 2013, 28 (6). 181-185.
19. Li Can, Rao Jingping, Li Shanju, et al. Application of Film Packaging in Postharvest Preservation of Fruits and Vegetables $[\mathrm{J}]$. Northern Horticulture, 2010(3):162-165.

20. Li Limei, Guan Junfeng, He Jingang et al. Effects of 1-MCP and MA on the Fruit Storage of Nanshui Pear [J]. Southwest China Journal of Agricultural Sciences, 2011, 24 (1): 240-243.

21. Wang Shihua. Effect of Packaging and Storage Temperature on the Storage of Lijiang Snow Peach [J]. Southwest China Journal of Agricultural Sciences, 2013, 26 (2): 697-700.

22. Zhu Yongqing, Yuan Huaiyu, Gao Jia, et al. Effects of Different Packaging Materials on the MAP-based storage of Toona sinensis [J]. Southwest China Journal of Agricultural Sciences, 2014, 27 (4): 1695-1699.

23. Wang Liqiong, Lin Shaohua, Chen Cunkun, et al. Effects of Three Different Preservation Methods on the Storage of Toona Sinensis [J]. Journal of Anhui Agricultural University, 2019, 46 (6): 1062-1068.

24. Yang Hui, Mao Weilin, Zhao Shouhuan, et al. Fresh-keeping of Toona Sinensis Buds Using Opening and Humidity Control Packaging at Ice Temperature [J]. Food and Machinery, 2017, 33 (9): 121-125.

25. Ma Jiajia, Huang Guili, Sui Siyao, et al. Effect of Microporous Packaging Combined with Low Temperature Plasma Sterilization on the Quality of Toona Sinensis on Shelf [J]. Journal of Food Safety and Quality Inspection, 2020, 11 (12): 3840-3846.

26. Zhao Xuehui. Research on the economic development of xiangzhu industry in Dazhu County. Modern Economic Information, 2019 (16): 502-502.

27. Li Changjie. The development of red incense in Dazhuba Mountain discusses [J]. China Forestry Economy, 2013(6). 